\title{
EL CUIDADO ENFERMERO EN LA ADMINISTRACIÓN SUBCUTÁNEA DE ENOXAPARINA EN PACIENTES ADULTOS: UNA REVISIÓN SISTEMATIZADA.
}

\author{
NURSING CARE IN THE \\ SUBCUTANEOUS ADMINISTRATION \\ OF ENOXAPARIN IN ADULT \\ PATIENTS: A SYSTEMATIC REVIEW
}

Recibido 18 de julio 2017 Aceptado 5 de septiembre 2017

Correspondencia: Mónica Margarita Lazo Sánchez Juan Badiano No. 1. Colonia Sección XVI Delegación Tlalpan. Código postal 14080 Teléfono: 55732911 extensión 1391 Correo electrónico: Izmonimar@gmail.com
Palabras clave: enoxaparina, inyecciones subcutáneas, heparina de bajo-peso-molecular, atención de enfermería.

Key words: enoxaparin, injections subcutaneous, heparin low-molecular-weight, nursing care.

\section{Autores:}

Mónica Margarita Lazo-Sánchez

Pasantía en Servicio Social.

Departamento de Investigación en Enfermería del Instituto Nacional de Cardiología Ignacio Chávez. Ciudad de México, México.

Julio César Cadena-Estrada Maestro en Enfermería.

Jefe de Educación e Investigación en Enfermería del Departamento de Investigación en Enfermería Instituto Nacional de Cardiología lgnacio Chávez. Ciudad de México, México. 


\section{RESUMEN}

Introducción: La enoxaparina ha provocado en el paciente cardiópata complicaciones por su mala administración; la probabilidad aumenta cuando se conjuga con otros factores de riesgo.

Objetivo: Analizar la evidencia sobre el cuidado de enfermería en la técnica de aplicación de enoxaparina.

Metodología: Estudio de revisión usando los pasos de la enfermería basada en evidencia: definición de la pregunta clínica, búsqueda con base en los términos DeCs en inglés, español y portugués, en las bases SciELO, LILACS, CUIDEN, Medline, REDALYC, CINAHL, y bidi UNAM. Se utilizó un instrumento constituido por: título, autor(es), año de publicación, idioma, área del conocimiento, nivel de evidencia, grado de recomendación, resultados y conclusión. El análisis de contenido generó tres dimensiones.

Resultados: El 69.7\% estabaeninglés; España generó más artículos (23.2\%); 53.4\% tenían nivel de evidencia IV/C. La dimensión factores de riesgo: relacionados con el paciente o tratamiento: polifarmacia e interacciones medicamentosas, >60-75 años, uso prolongado, diagnóstico cardiológico. La técnica de administración: velocidad lenta, esperando antes de retirar la aguja. Finalmente, complicaciones: cómo hematomas abdominales, necrosis cutánea.

Conclusión: El cuidado enfermero en la administración de enoxaparina debe basarse en una adecuada valoración que permita identificar factores de riesgo y reducir la probabilidad de complicaciones.

Palabras clave: enoxaparina, inyecciones subcutáneas, heparina de bajo-peso-molecular, atención de enfermería.

\section{ABSTRACT}

Introduction: Enoxaparin has caused cardiac complications due to poor administration; the probability increases when combined with other risk factors.

Objective: Analyze the evidence of nursing care in the application technique of enoxaparin.

Methodology: Review using evidence based nursing steps: definition of the clinical question, search based on terms DeCS in English, Spanish and Portuguese, in the databases of SciELO, LILACS, CUIDEN, Medline, REDALYC, CINAHL, and bidi UNAM. We used an instrument constituted by: title, author(s), year of publication, language, the area of knowledge, level of evidence, the degree of recommendation, results and conclusion. The content analysis generated three dimensions.

Results: $69.7 \%$ published in English; Spain generated more articles (23.2\%); 53.4\% had level of evidence IV/C. The dimension risk factors: patient-related or treatmentrelated: polypharmacy and drug interactions, >6075 years, prolonged use, cardiologic diagnosis. The technique of administration: slowly speed, waiting before withdrawing the needle. Finally, complications: such as hematomas of the abdominal wall, cutaneous necrosis.

Conclusion: Nursing care in the administration of enoxaparin should be based on an adequate assessment to identify risk factors and reduce the likelihood of complications.

Key words: enoxaparin, injections subcutaneous, heparin low-molecular-weight, nursing care.
106

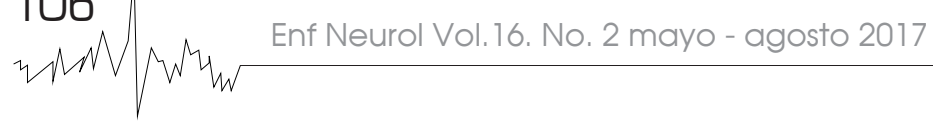




\section{INTRODUCCIÓN}

$\square$

n enfermería la administración de medicamentos es un actividad básica de cuidado y

una responsabilidad muy importante en su quehacer diario, que incluso se encuentra normado por el Consejo de Salubridad General para la Atención en Salud con Calidad y Seguridad y dentro de las Metas Internacionalesde Seguridad del Paciente (MISP), específicamente en la número 3 "Mejorar la seguridad de los medicamentos de alto riesgo", enfocada a prevenir eventos adversos derivados de la atención en la administración de medicamentos, tales como los electrolitos concentrados, quimioterapéuticos, radiofármacos, insulinas y anticoagulantes? los cuales son el tema central de este estudio.

Las heparinas de bajo peso molecular (HBPM) son un tipo de anticoagulante que se prepara a partir de la heparina no fraccionada, ésta es extraída de la mucosa intestinal porcina o de pulmones bovinos a través de la despolimerización química - enzimática. Una vez administrada se excreta por vía renal; y mientras más pequeño es el peso molecular, más lenta es su eliminación, como en el caso de la enoxaparina. ${ }^{2}$

La acción de esta HBPM requiere de la antitrombina III, capaz de neutralizar a la trombina mediante su acción anticoagulante y para lo que se necesitan 18 sacáridos. Tienetambién una acción antitrombótica, que mediante la unión a la antitrombina III, inhibe el factor Xa y sólo necesita cinco sacáridos. Por esta razón posee una mayor capacidad antitrombótica que anticoagulante, con una proporción 3:1 en el caso de la enoxaparina. ${ }^{3.4}$ La inhibición del factor Xa por las HBPM en general, induce cambios muy discretos en el tiempo de tromboplastina parcial, es por esa razón, que no es conveniente basarse en los resultados de este estudio para su control. ${ }^{5}$

La enoxaparina ha ido desplazando, en los últimos años, a la heparina no fraccionada por sus mejores propiedades farmacodinámicas, además de por su mayor seguridady comodidad en la administración. Sin embargo, a pesar de sus numerosos beneficios, se han reportado casos de complicaciones locales por su mal uso al momento de aplicarlas, que pueden ocasionar complicaciones que van desde dolor y aparición de hematomas, hasta necrosis cutáneas.

En las instituciones de salud, los investigadores han encontrado factores como el uso de jeringas precargadas, el purgado de la burbuja de aire en éstas, la aspiración antes de la inyección, la aplicación de frío o calor en el sitio de inyección, la velocidad durante la administración y el tiempo para poder retirar la aguja después de la inyección, como aquellos que influyen en la aparición de complicaciones. Aunado a esto se ha encontrado, en los estudios, que la probabilidad de desarrollar complicaciones es mayor cuando los pacientes están tomando otros fármacos, tienen un índice de masa corporal inferior o superior al normal, tienen enfermedad renal crónica o son mayores de 65 años. Además del daño físico que pueden ocasionar, también traen consigo un incremento en los días de estancia hospitalaria y el costo de los recursos necesarios para su tratamiento y rehabilitación.

En México existen pocos estudios que unifiquen el método adecuado para la administración de enoxaparina, específicamente en el sitio, volumen de inyección y las intervenciones para la reducción de complicaciones locales; y los existentes muestran cierta variabilidad. Por lo tanto, las técnicas deben ser examinadas y probadas para promover una mayor comodidad del paciente y mejorar su calidad de vida evitando el desarrollo de reacciones locales o, incluso, la pérdida de la confianza del paciente en el personal de salud para posteriores administraciones. Es por ello que el presente trabajo busca analizar la evidencia científica sobre el cuidado de enfermería en la técnica de aplicación de la enoxaparina.

Con base en los resultados se pretende aportar información a la comunidad docente, estudiantil y asistencial, ya que de esta manera se podrán emprender acciones concretas en cuanto a su uso y técnica. Además, estos hallazgos pueden dar pauta para llevar a cabo estudios de investigación, en un futuro, para poder determinar qué técnica aceptar, o rechazar, en la práctica clínica.

Enf Neurol Vol. 16. No. 2 mayo - agosto 2017 . 


\section{METODOLOGÍA}

S e realizó un estudio de revisión mediante la metodología integradora, bajo el enfoque cualitativo y descriptivo, realizado con evidencia nacional e internacional, sobre la administración de la enoxaparina en el paciente adulto, para lo cual se siguieron los pasos de la enfermería basada en la evidencia: selección del tema y definición de la pregunta clínica, búsqueda de la mejor evidencia disponible, categorización de los estudios, evaluación crítica, interpretación de los resultados y presentación de la revisión integradora. Se planteó la siguiente pregunta clínica que guió el estudio: ¿Cuál es la mejor técnica de inyección subcutánea para la administración de enoxaparina en pacientes adultos?

Se realizó la búsqueda de la evidencia en las bases de datos de SciELO, LILACS, CUIDEN, Medline, REDALYC, CINAHL, y en la biblioteca de la UNAM. Una de las estrategias utilizadas fue el uso de los Descriptores en Ciencias de la Salud (DeCS) "enoxaparina", "adulto", "inyecciones subcutáneas" y "heparina de bajo-pesomolecular" en español, inglés y portugués; junto con el operador boleano "AND".

Con la finalidad de identificar la evidencia potencialmente relevante para el estudio, se realizó una lectura crítica, rápida y detallada de los títulos y resúmenes; incluyendo aquellos artículos científicos en texto completo, idioma inglés, español y portugués. Al no encontrar numerosos artículos en los últimos cinco años, se amplió la búsqueda a aquellos publicados del 01 de Enero de 2005 al 31 de diciembre de 2016 y, se excluyeron del análisis los textos que no estaban relacionados con el tema, en los que sólo estaba disponible el resumen y no el texto completo y los que estuvieron repetidos en otras bases de datos.

Para una mejor lectura de las publicaciones que constituyen el corpus del estudio, se utilizó un instrumento ex profeso constituido por las variables: título, autor(es), año de publicación, revista, país de origen, idioma, disponibilidad, área del conocimiento, objetivo del estudio, material y métodos (tipo y diseño, tamaño de la población y muestra, criterios de selección, instrumento e intervención), nivel de evidencia, grado de recomendación, principales hallazgos y conclusión.

El nivel de evidencia es presentado de acuerdo a la US Agency for Health care Research and Quality con modificación del grado de recomendación por las Scottish Intercollegiate Guidelines Network.

Para el análisis y la interpretación de los datos a profundidad se utilizó como referencia el análisis de contenido (observación, investigación de materiales, hallazgos y conclusiones)', obteniendo como resultado tres categorías: factores de riesgo, técnica de inyección y complicaciones.

\section{RESULTADOS}

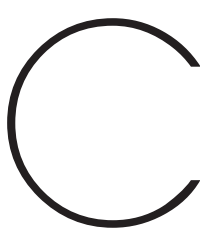
on base en los pasos de la revisión sistematizada con metodología integradora, se lograron identificar 13,126 artículos de las seis bases de datos de donde se realizó la búsqueda electrónica. No obstante, sólo 43 artículos científicos cumplieron con los criterios de selección, mismos que constituyen el cuerpo de este estudio (Cuadro I).

Prevalecieron los estudios de caso (34.9\%), seguido de los ensayos clínicos aleatorizados (13.9\%), los estudios descriptivos/observacionales (13.9\%) y los estudios de revisión (sistematizada) (13.9\%) (Figura 1).

El inglés fue el idioma en el cual se escribieron la mayoría de los artículos (69.7\%), seguido del español (27.9\%). España fue el país de donde se generaron más artículos (23.2\%), seguido de Turquía y EUA (16.2\%); y en relación al área del conocimiento el 51. $1 \%$ eran del área médica.

Del total de los artículos analizados, la mayoría tenían un nivel de evidencia IV/C (53.4\%), seguidos de los niveles Ib/A y III/C (13.9\%). En el año 2013 se publicaron más artículos (16.2\%), seguido del 2007 (13.9\%), 2017 y 2016 (11.6\%).

\section{8} MMN $\mathrm{WH}_{W}$ Enf Neurol Vol. 16. No. 2 mayo - agosto 2017 
Del análisis de contenido a profundidad se generaron tres dimensiones (Cuadro II); la primera fue acerca de los factores de riesgo: 1) relacionados con el paciente, como la polifarmacia e interacciones medicamentosas (antiagregantes, anticoagulantes, fibrinolíticos), ${ }_{1}^{10 / 12} \mathrm{MC}>25 \mathrm{~kg} / \mathrm{m}^{2,12 / 14}$ género femenino, $14-16$ edad >60-75 años,12,15/17 insuficiencia renal grave $($ ACr $<30 \mathrm{ml} / \mathrm{min}),{ }^{14,15}$ tensión abdominal (tos), ${ }^{14}$ poca grasa abdominal; ${ }^{17-20}$ y 2) los relacionados con el tratamiento, como el uso de enoxaparina genérica, ${ }^{27}$ en cateterismo cardiaco, ${ }^{12}$ diagnóstico cardiológico, ${ }^{12 / 18}$ artroplastial22 y tratamiento prolongado (>9 días). ${ }^{13 / 16}$

La segunda dimensión fue acerca de la técnica de la administración, ésta debe ser con jeringas precargadas, ${ }^{13 / 25}$ con burbuja de aire, sin aspirado previo, ${ }^{25 / 27}$ a una velocidad lenta ${ }^{28 / 35}$ esperando antes de retirar la aguja, ${ }^{31,32}$ se puede aplicar compresas calientes o frías si hubo ocurrencia de hematomas. ${ }^{34 / 37}$

Finalmente, la dimensión de complicaciones relacionada con la administración como los hematomas de la pared abdominal, ${ }^{17,20,38 / 47}$ el dolor, ${ }^{19}$ la dermatosis ampollosa,42 la ulceración de la piel y tejido subcutáneo; 43 en estos casos se puede tratar con un manejo conservador mediante reposo en cama, manejo del dolor, monitorización, suspensión de la enoxaparina y valoración de un posible uso de un anticoagulante alternativo. ${ }^{40.44 .45}$ También se pueden presentar hemorragias retroperitoneales ${ }^{46}$ y necrosis cutánea. ${ }^{47,48}$
Por lo tanto, con base en la evidencia se recomienda que el profesional de enfermería haga una valoración de los potenciales factores de riesgo del paciente (Illo/B-IV/C) o del tratamiento (Illb/B-IV/C) y, una vez identificando la existencia de éstos, se debe ajustar la dosis y monitorizar los niveles de anti-Xa (IV/C), cabe mencionar que dentro de esta valoración se tiene que evaluar la técnica de administración, y en caso de que exista deficiencia es importante llevar a cabo los siguientes pasos: colocar al paciente en posición supina, seleccionar el flanco a puncionar, realizar la asepsia del sitio, preferentemente con jeringas precargas (III/C-lb/A), realizar elevación del pliegue cutáneo, no se debe purgar la burbuja de aire (IV/C) ni realizarse aspirado (Ib/A-IV/C) al momento de la aplicación, administrar a una velocidad lenta entre 15-30 segundos (la/AIV/C), esperar 5-10 segundos para retirar la aguja (lla/B) y colocar paquetes de calor seco o fríocaliente en el sitio si hay aparición de hematomas (lb/A-lla/B). Evaluar posteriormente, en caso de que la técnica sea la correcta o no, si hubo aparición de complicaciones leves/moderadas - graves y dar el tratamiento correspondiente (IV/C). Si se desarrollaron o no complicaciones y, en caso de que se hayan resuelto las mismas, se debe mantener al paciente bajo vigilancia y observación buscando siempre la mejora en su bienestar, que él aprenda a identificar signos/ síntomas de alarma y que el profesional de enfermería conozca la técnica de administración correcta (Fig. 2). 
Figura 1. Búsqueda Sistematizada de la Evidencia sobre la administración de enoxaparina en adultos

$\mathrm{N}=13,126$

Identificados por búsqueda electrónica

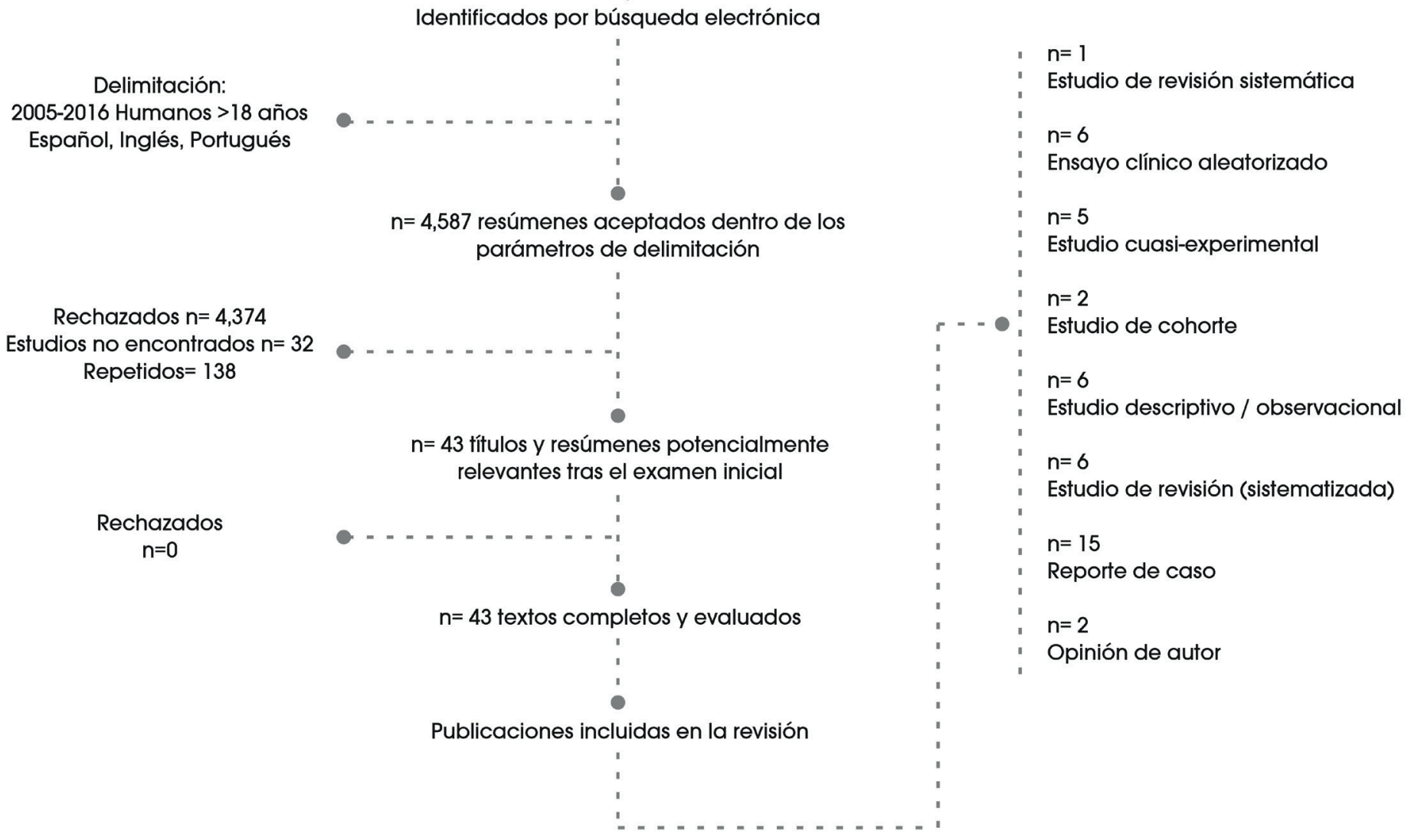


Figura 2. Cuidado de enfermería en la administración de enoxaparina en el paciente adulto hospitalizado. ( Parte 1)

\section{Administración subcutánea de enoxaparina en adultos}

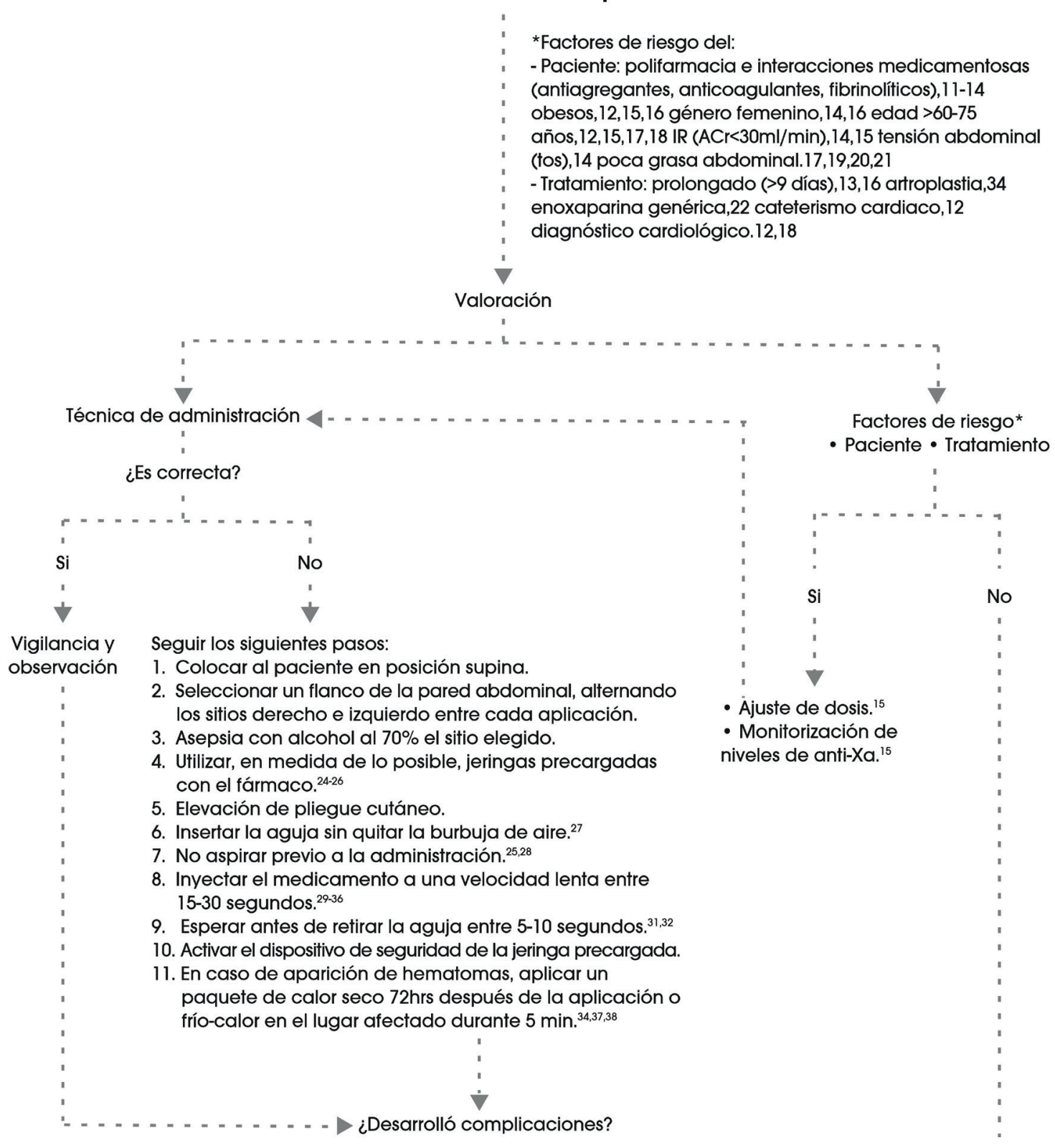


Figura 2. Cuidado de enfermería en la administración de enoxaparina en el paciente adulto hospitalizado. ( Parte 2)

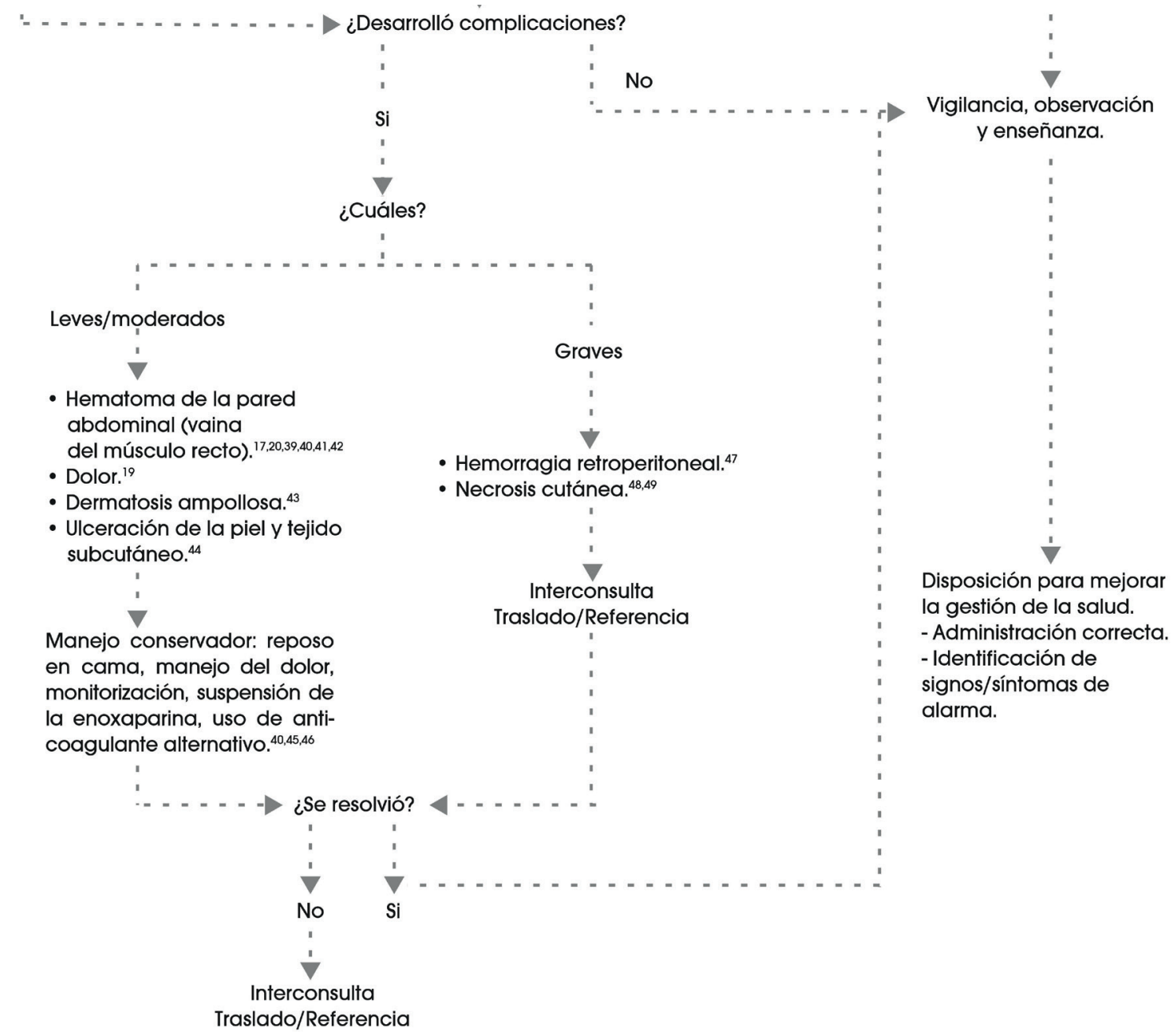


Disposición para mejorar la gestión de la salud

- Administración correcta

- Identificación de signos/síntomas de alarma

\section{Cuadro 1. Síntesis de la evidencia relacionada con la administración de enoxaparina en adultos.}

\begin{tabular}{|c|c|c|c|c|c|c|c|c|}
\hline Autor & País & Idioma & Metodología & NE/GR & Muestra & Patología & Variables & Resultados \\
\hline Rangel CR & Colombia & Español & $\begin{array}{l}\text { Descriptivo/ } \\
\text { Observacional }\end{array}$ & $\| I I / C$ & $\begin{array}{c}284 \\
\text { pacientes }\end{array}$ & & $\begin{array}{l}\text { RAM (reacciones } \\
\text { adversas } \\
\text { amedicamentos) }\end{array}$ & $\begin{array}{l}\text { Los factores asociados para la } \\
\text { aparición de RAM son la } \\
\text { polifarmacia y las interacciones } \\
\text { medicamentosas. }\end{array}$ \\
\hline $\begin{array}{l}\text { Manresa- } \\
\text { Ramón N. }\end{array}$ & España & Español & $\begin{array}{c}\text { De revisión } \\
\text { (sistematizada) }\end{array}$ & $\mathrm{IV} / \mathrm{C}$ & $\begin{array}{l}\text { Meta análisis, } \\
\text { ensayos } \\
\text { clínicos, seguidos } \\
\text { de estudios de } \\
\text { casos, de últimos } \\
10 \text { años }\end{array}$ & & $\begin{array}{l}\text { 1. Insuficiencia renal } \\
\text { grave }(\mathrm{ACr}<30 \mathrm{ml} / \mathrm{min}) \\
\text { 2. Edad avanzada }>75 \\
\text { años) } \\
\text { 3. Px. obesos } \\
\text { (IMC }>40 \mathrm{~kg} / \mathrm{m} 2) \\
\text { 4. Embarazadas }\end{array}$ & $\begin{array}{l}\text { 1. Monitorización anti-Xa, dosis de } \\
20 \mathrm{mg} / 24 \mathrm{hr} \text {. } \\
\text { 2. Dosis de } 1 \mathrm{mg} / \mathrm{kg} / 12 \mathrm{hr} \text { o. } 75 \text { si tuvo } \\
\text { IAMCEST y monitorización. } \\
\text { 3. } 1 \mathrm{mg} / \mathrm{kg}(\text { peso real)/12hr, } \\
\text { monitorización anti-Xa. } \\
4.1 \mathrm{mg} / \mathrm{kg} / 12 \mathrm{~h} \text { y monitorizar los } \\
\text { niveles anti-Xa. }\end{array}$ \\
\hline Pierri H. & Brasil & Portugués & Reporte de caso & $\mathrm{IV} / \mathrm{C}$ & $\begin{array}{c}1 \\
\text { paciente }\end{array}$ & $\begin{array}{l}\text { Enfermedad } \\
\text { de las } \\
\text { arterias } \\
\text { coronarias }\end{array}$ & $\begin{array}{l}\text { Hematoma en la } \\
\text { pared abdominal } \\
\text { hasta el retroperitoneo }\end{array}$ & $\begin{array}{l}\text { Se le realizó una laparotomía de } \\
\text { emergencia para eliminar el } \\
\text { hematoma. }\end{array}$ \\
\hline Gomes M. & EUA & Español & $\begin{array}{l}\text { Opinión de un } \\
\text { autor }\end{array}$ & $\mathrm{IV} / \mathrm{C}$ & & & $\begin{array}{l}\text { Enoxaparina genérica } \\
\text { en SCA }\end{array}$ & $\begin{array}{l}\text { El uso de enoxaparina genérica en } \\
\text { indicaciones como SCA debe ser } \\
\text { validado antes de su aprobación. }\end{array}$ \\
\hline Tomás-Rojas J & España & Español & $\begin{array}{c}\text { De revisión } \\
\text { (sistematizada) }\end{array}$ & IV/C & $\begin{array}{c}45 \\
\text { profesionales }\end{array}$ & & $\begin{array}{l}\text { Burbuja de aire en las } \\
\text { jeringas precargadas }\end{array}$ & $\begin{array}{l}\text { Inyectar la burbuja de aire busca } \\
\text { tres efectos: evitar la pérdida de } \\
\text { medicamento, favorecer el sellado, } \\
\text { evitar medicación no deseada en } \\
\text { capas más superficiales. }\end{array}$ \\
\hline Lamblet LCR & Brasil & Español & $\begin{array}{l}\text { Ensayo clínico } \\
\text { aleatorizado }\end{array}$ & $\mathrm{Ib} / \mathrm{A}$ & $\begin{array}{l}240 \text { pacientes } \\
\text { para inyección } \\
\text { SC }\end{array}$ & & $\begin{array}{l}\text { Jeringa de aguja fija } \\
\text { retráctil y con cambio } \\
\text { de aguja }\end{array}$ & $\begin{array}{l}\text { El uso de jeringa con dispositivo de } \\
\text { seguridad retráctil con aguja fija no } \\
\text { compromete la sensación de dolor } \\
\text { ni aumenta el riesgo de formación } \\
\text { de hematomas. }\end{array}$ \\
\hline Gómez MJ & España & Español & $\begin{array}{l}\text { Ensayo clínico } \\
\text { aleatorizado }\end{array}$ & $\mathrm{Ib} / \mathrm{A}$ & $\begin{array}{c}297 \\
\text { pacientes }\end{array}$ & & $\begin{array}{l}\text { Jeringa cargada por el } \\
\text { profesional con y sin } \\
\text { aspirado. Jeringa } \\
\text { precargada con y } \\
\text { sin aspirado }\end{array}$ & $\begin{array}{l}\text { Se establece la disminución de } \\
\text { lesiones con la utilización del } \\
\text { fármaco en jeringas precargadas. } \\
\text { No hay conclusiones sobre la } \\
\text { técnica de aspirado previo. }\end{array}$ \\
\hline Cerrillo-Patiño JR & España & Español & $\begin{array}{l}\text { Descriptivo/ } \\
\text { Observacional }\end{array}$ & III/C & & & $\begin{array}{l}\text { Técnica de aspirado o } \\
\text { no previo a la } \\
\text { administración y } \\
\text { posición del paciente }\end{array}$ & $\begin{array}{l}\text { Investigar la variabilidad de efectos } \\
\text { locales de cada HBPM, la aparición } \\
\text { de lesiones en diferentes zonas } \\
\text { anatómicas, la posición del } \\
\text { paciente, si debe quedar en reposo } \\
\text { posterior a la administración. }\end{array}$ \\
\hline $\begin{array}{l}\text { Rodríguez- } \\
\text { Gallego I }\end{array}$ & España & Español & $\begin{array}{c}\text { De revisión } \\
\text { (sistematizada) }\end{array}$ & IV/C & $\begin{array}{c}17 \\
\text { artículos }\end{array}$ & & $\begin{array}{l}\text { Técnica con y sin } \\
\text { aspirado previo }\end{array}$ & $\begin{array}{l}\text { Existen pocos estudios sobre la } \\
\text { relación entre la supresión del paso } \\
\text { de aspirado previo y la aparición } \\
\text { de hematomas abdominales. }\end{array}$ \\
\hline Alcahúd CC & España & Español & $\begin{array}{l}\text { Estudio de } \\
\text { cohortes }\end{array}$ & $\mathrm{llb} / \mathrm{B}$ & $\begin{array}{c}172 \\
\text { pacientes }\end{array}$ & & $\begin{array}{l}\text { Factores de riesgo } \\
\text { relacionados con la } \\
\text { aparición de } \\
\text { hematomas }\end{array}$ & $\begin{array}{l}\text { Los factores de riesgo relacionados } \\
\text { fueron: obesidad, adultos mayores, } \\
\text { diagnóstico cardiológico, } \\
\text { antiagregantes, fibrinolíticos, } \\
\text { anticoagulantes y cateterismo } \\
\text { cardiaco. }\end{array}$ \\
\hline
\end{tabular}




\begin{tabular}{|c|c|c|c|c|c|c|c|c|}
\hline Autor & País & Idioma & Metodología & NE/GR & Muestra & Patología & Variables & Resultados \\
\hline Cerrillo-Patiño JR & España & Español & $\begin{array}{l}\text { Descriptivo/ } \\
\text { Observacional }\end{array}$ & $\mathrm{III} / \mathrm{C}$ & & & $\begin{array}{l}\text { Conocimientos y } \\
\text { prácticas de } \\
\text { enfermería sobre } \\
\text { la técnica }\end{array}$ & $\begin{array}{l}\text { Tienen conocimientos sobre la } \\
\text { técnica de administración; el } \\
\text { tiempo de ejercicio profesional es } \\
\text { un factor de riesgo para una } \\
\text { inadecuada técnica; las jeringas } \\
\text { precargadas reducen errores en } \\
\text { la dosificación. }\end{array}$ \\
\hline Firoozbakhsh S & Irán & Inglés & Reporte de caso & $\mathrm{IV} / \mathrm{C}$ & $\begin{array}{c}1 \\
\text { paciente }\end{array}$ & EPOC & $\begin{array}{l}\text { Hematoma en la vaina } \\
\text { del músculo recto del } \\
\text { abdomen }\end{array}$ & $\begin{array}{l}\text { La mayoría de los casos pueden ser } \\
\text { manejados de forma conservadora } \\
\text { con reposo en cama y manejo del } \\
\text { dolor; el paciente debe estar } \\
\text { monitorizado; o cirugía con } \\
\text { hemostasia o ligadura de la arteria } \\
\text { inferior epigástrica. }\end{array}$ \\
\hline Rahman MS & Reino Unido & Inglés & Reporte de caso & $\mathrm{IV} / \mathrm{C}$ & $\begin{array}{c}1 \\
\text { paciente }\end{array}$ & IAMSEST & $\begin{array}{l}\text { Hematoma } \\
\text { subcutáneo masivo en } \\
\text { la pared torácica }\end{array}$ & $\begin{array}{l}\text { Se le aplicó un vendaje a presión } \\
\text { de acuerdo con una revisión } \\
\text { quirúrgica vascular del consultor; } \\
\text { requirió de transfusiones } \\
\text { sanguíneas. }\end{array}$ \\
\hline Ivanova I & España & Español & Reporte de caso & $\mathrm{IV} / \mathrm{C}$ & $\begin{array}{c}1 \\
\text { paciente }\end{array}$ & $\begin{array}{l}\text { Fractura de } \\
\text { peroné } \\
\text { izquierdo con } \\
\text { tratamiento } \\
\text { quirúrgico }\end{array}$ & $\begin{array}{l}\text { Lesiones cutáneas en } \\
\text { abdomen centradas } \\
\text { en cada punto de } \\
\text { inoculación del } \\
\text { fármaco }\end{array}$ & $\begin{array}{l}\text { Suspender la heparina implicada, si } \\
\text { las lesiones son localizadas se } \\
\text { puede sustituir por otra HBPM, si las } \\
\text { lesiones son diseminadas no. }\end{array}$ \\
\hline Yusuf A & Turquía & Inglés & Reporte de caso & $\mathrm{IV} / \mathrm{C}$ & $\begin{array}{c}1 \\
\text { paciente }\end{array}$ & SCA & $\begin{array}{l}\text { Dolor severo en la } \\
\text { región abdominal } \\
\text { inferior izquierda }\end{array}$ & $\begin{array}{l}\text { Las causas pueden ser una } \\
\text { inyección accidental por vía } \\
\text { intramuscular, la disrupción de los } \\
\text { vasos epigástricos. El uso de HBPM } \\
\text { requiere un seguimiento en } \\
\text { aquellos pacientes con poca grasa } \\
\text { abdominal. }\end{array}$ \\
\hline Toll A & España & Inglés & Reporte de caso & $\mathrm{IV} / \mathrm{C}$ & $\begin{array}{c}1 \\
\text { paciente }\end{array}$ & $\begin{array}{l}\text { HAS, DM, } \\
\text { hiperuricemia, } \\
\text { FA, bronquitis } \\
\text { crónica y } \\
\text { policitemia }\end{array}$ & $\begin{array}{l}\text { Trombos de fibrina en } \\
\text { la microvasculatura } \\
\text { dérmica con necrosis } \\
\text { isquémica de la } \\
\text { epidermis superpuesta }\end{array}$ & $\begin{array}{l}\text { Se cree que la necrosis cutánea es } \\
\text { causada por una condición } \\
\text { protrombótica local mediada por } \\
\text { anticuerpos asociada con la } \\
\text { activación plaquetaria y una } \\
\text { producción incrementada de } \\
\text { trombina. }\end{array}$ \\
\hline Kumar DA & Reino Unido & Inglés & Reporte de caso & $\mathrm{IV} / \mathrm{C}$ & $\begin{array}{c}1 \\
\text { paciente }\end{array}$ & $\begin{array}{l}\text { Angina de } \\
\text { pecho }\end{array}$ & $\begin{array}{l}\text { Induración de la piel y } \\
\text { grasa subcutánea de } \\
\text { la mama izquierda }\end{array}$ & $\begin{array}{l}\text { Las HBPM pueden causar necrosis } \\
\text { grasa de la mama, un } \\
\text { anticoagulante alternativo debe } \\
\text { ser usado en pacientes afectados. }\end{array}$ \\
\hline Schindewolf M & Alemania & Inglés & $\begin{array}{l}\text { Estudio de } \\
\text { cohortes }\end{array}$ & $\mathrm{llb} / \mathrm{B}$ & $\begin{array}{c}320 \\
\text { pacientes }\end{array}$ & & $\begin{array}{l}\text { Incidencia de lesiones } \\
\text { cutáneas }\end{array}$ & $\begin{array}{l}\text { Se identificó: un índice de masa } \\
\text { corporal superior a } 25 \text {, duración de } \\
\text { la terapia mayor a } 9 \text { días, sexo } \\
\text { femenino como factores de riesgo. }\end{array}$ \\
\hline Palese A & Italia & Inglés & $\begin{array}{c}\text { Estudio } \\
\text { Cuasi-experimental }\end{array}$ & $\mathrm{llb} / \mathrm{B}$ & $\begin{array}{c}150 \\
\text { pacientes }\end{array}$ & & $\begin{array}{l}\text { Duración de la } \\
\text { inyección sobre la } \\
\text { ocurrencia y extensión } \\
\text { de hematomas }\end{array}$ & $\begin{array}{l}\text { La administración subcutánea lenta } \\
\text { de HBPM (de al menos } 30 \text { s) reduce } \\
\text { la aparición de hematomas. }\end{array}$ \\
\hline $\begin{array}{c}\text { Sobieszczańska } \\
\text { M }\end{array}$ & Polonia & Inglés & $\begin{array}{l}\text { Reporte } \\
\text { de caso }\end{array}$ & IV/C & $\begin{array}{c}1 \\
\text { paciente }\end{array}$ & $\begin{array}{l}\text { IAM y } \\
\text { embolismo } \\
\text { pulmonar }\end{array}$ & $\begin{array}{l}\text { Ulceración de la piel y } \\
\text { tejido subcutáneo en } \\
\text { ambos lados del } \\
\text { abdomen }\end{array}$ & $\begin{array}{l}\text { La administración de Flucinar } \mathrm{N} \\
\text { resultó en la regresión de los } \\
\text { síntomas. }\end{array}$ \\
\hline Visvanathan V & Australia & Inglés & $\begin{array}{l}\text { Revisión } \\
\text { sistemática }\end{array}$ & $\mathrm{la} / \mathrm{A}$ & $\begin{array}{l}50 \\
\text { pacientes de } \\
\text { un ECA }\end{array}$ & & $\begin{array}{l}\text { Duración de la } \\
\text { inyección en relación } \\
\text { al dolor y aparición de } \\
\text { hematomas }\end{array}$ & $\begin{array}{l}\text { La administración subcutánea lenta } \\
\text { resultó en menor intensidad del } \\
\text { dolor y menor tamaño del } \\
\text { hematoma en el sitio de inyección. }\end{array}$ \\
\hline Parkinson $\mathrm{F}$ & Reino Unido & Inglés & $\begin{array}{l}\text { Reporte } \\
\text { de caso }\end{array}$ & IV/C & $\begin{array}{c}1 \\
\text { paciente }\end{array}$ & $\begin{array}{l}\text { Gangrena del } \\
\text { pie izquierdo }\end{array}$ & $\begin{array}{l}\text { Hematoma en la } \\
\text { pared abdominal } \\
\text { anterior }\end{array}$ & $\begin{array}{l}\text { El mecanismo directo podría ser la } \\
\text { inyección intramuscular inadvertida } \\
\text { o la interrupción de los vasos } \\
\text { epigástricos. }\end{array}$ \\
\hline
\end{tabular}




\begin{tabular}{|c|c|c|c|c|c|c|c|c|}
\hline Autor & País & Idioma & Metodología & NE/GR & Muestra & Patología & Variables & Resultados \\
\hline Loidi PL & España & Español & $\begin{array}{l}\text { Reporte } \\
\text { de caso }\end{array}$ & $\mathrm{IV} / \mathrm{C}$ & $\begin{array}{c}2 \\
\text { pacientes }\end{array}$ & $\begin{array}{l}\text { EPOC y EVC } \\
\text { isquémico }\end{array}$ & $\begin{array}{l}\text { Vesículo-ampollas } \\
\text { hemorrágicas }\end{array}$ & $\begin{array}{l}\text { La dermatosis ampollosa } \\
\text { hemorrágica se caracteriza por la } \\
\text { aparición de éstas en lugares } \\
\text { distantes al lugar de inyección. }\end{array}$ \\
\hline Uzun S & Turquía & Inglés & $\begin{array}{c}\text { Estudio } \\
\text { cuasi-experimental }\end{array}$ & Ila/B & $\begin{array}{c}48 \\
\text { pacientes }\end{array}$ & & $\begin{array}{l}\text { Duración de la } \\
\text { inyección y retirada } \\
\text { de la aguja }\end{array}$ & $\begin{array}{l}\text { La formación de equimosis y el } \\
\text { tamaño disminuyen con un tiempo } \\
\text { de administración mayor ( } 30 \text { s), y la } \\
\text { retirada tardía de la aguja (10s) } \\
\text { puede disminuir aún más el } \\
\text { tamaño del moretón. }\end{array}$ \\
\hline Kayrak M & Turquía & Inglés & $\begin{array}{l}\text { Reporte } \\
\text { de caso }\end{array}$ & $\mathrm{IV} / \mathrm{C}$ & $\begin{array}{c}1 \\
\text { paciente }\end{array}$ & $\begin{array}{l}\text { Dificultad } \\
\text { respiratoria }\end{array}$ & Hematoma & $\begin{array}{l}\text { Las inyecciones subcutáneas de la } \\
\text { pared abdominal tal vez } \\
\text { incrementen el riesgo de } \\
\text { hematoma, especialmente en } \\
\text { pacientes mayores y delgados con } \\
\text { menos grasa abdominal. }\end{array}$ \\
\hline Amaniyan S & Irán & Inglés & $\begin{array}{l}\text { Ensayo clínico } \\
\text { aleatorizado }\end{array}$ & $\mathrm{Ib} / \mathrm{A}$ & $\begin{array}{c}60 \\
\text { pacientes }\end{array}$ & $\begin{array}{l}\text { Enfermedad } \\
\text { coronaria }\end{array}$ & $\begin{array}{l}\text { Aplicación de } \\
\text { paquetes locales de } \\
\text { frío y calor-frío }\end{array}$ & $\begin{array}{l}\text { La aplicación de paquetes locales } \\
\text { fríos y calientes conduce a una } \\
\text { disminución en el tamaño y la } \\
\text { frecuencia de moretones después } \\
\text { de las inyecciones de } 60 \mathrm{mg} \text { de } \\
\text { enoxaparina sódica. Además, la } \\
\text { aplicación combinada de frío y } \\
\text { calor local mejoró la eficacia de la } \\
\text { intervención. }\end{array}$ \\
\hline Black L & EUA & Inglés & $\begin{array}{c}\text { De revisión } \\
\text { (sistematizada) }\end{array}$ & $\mathrm{IV} / \mathrm{C}$ & $\begin{array}{c}2,402 \\
\text { lesiones }\end{array}$ & & $\begin{array}{l}\text { Eliminación de la } \\
\text { práctica de elevación } \\
\text { manual del pliegue } \\
\text { cutáneo }\end{array}$ & $\begin{array}{l}\text { La implementación de la longitud } \\
\text { apropiada de la aguja y la técnica } \\
\text { de inyección alternadapermite que } \\
\text { la medicación sea entregada en el } \\
\text { tejido subcutáneo sin exponer a los } \\
\text { pacientes ni a los proveedores de } \\
\text { atención a lesiones evitables. }\end{array}$ \\
\hline Rushing J & EUA & Inglés & $\begin{array}{l}\text { Opinión de } \\
\text { un autor }\end{array}$ & $\mathrm{IV} / \mathrm{C}$ & & & $\begin{array}{l}\text { Técnica en general } \\
\text { de inyección }\end{array}$ & $\begin{array}{l}\text { Lo que se debe y no se debe de } \\
\text { hacer al aplicar una inyección. }\end{array}$ \\
\hline Pourghaznein T & Irán & Inglés & $\begin{array}{c}\text { Estudio } \\
\text { cuasi-experimental }\end{array}$ & $\| \mathrm{la} / \mathrm{B}$ & $\begin{array}{c}90 \\
\text { pacientes }\end{array}$ & & $\begin{array}{l}\text { Duración de la } \\
\text { inyección y retirada } \\
\text { de la aguja }\end{array}$ & $\begin{array}{l}15 \text { segundos de duración de la } \\
\text { inyección y esperar por } 5 \text { segundos } \\
\text { antes de retirar la aguja es } \\
\text { recomendado en la inyección de } \\
\text { heparina subcutánea. La severidad } \\
\text { del dolor en el abdomen es menor } \\
\text { que en los muslos. }\end{array}$ \\
\hline Balci AR & Turquía & Inglés & $\begin{array}{c}\text { Estudio } \\
\text { cuasi-experimental }\end{array}$ & $\| \mathrm{la} / \mathrm{B}$ & $\begin{array}{c}33 \\
\text { pacientes }\end{array}$ & & $\begin{array}{l}\text { Aplicación de paquete } \\
\text { de calor seco local }\end{array}$ & $\begin{array}{l}\text { Se recomienda la aplicación de } \\
\text { un paquete de calor seco local } \\
72 \text { horas después de la última } \\
\text { inyección subcutáneapara } \\
\text { acelerar la recuperación de } \\
\text { hematomas. }\end{array}$ \\
\hline Zaybak A & Turquía & Inglés & $\begin{array}{c}\text { Estudio } \\
\text { cuasi-experimental }\end{array}$ & $\| \mathrm{la} / \mathrm{B}$ & $\begin{array}{c}50 \\
\text { pacientes }\end{array}$ & & $\begin{array}{l}\text { Duración de la } \\
\text { inyección y factores } \\
\text { demográficos }\end{array}$ & $\begin{array}{l}\text { No hubo correlación entre la edad } \\
\text { o el grosor de tejido subcutáneo y } \\
\text { el tamaño de moretones, la } \\
\text { intensidad del dolor y la duración } \\
\text { del dolor. Se apoya el uso de } 30 \text { s } \\
\text { durante la administración. }\end{array}$ \\
\hline Sendir M & Turquía & Inglés & $\begin{array}{l}\text { Ensayo clínico } \\
\text { aleatorizado }\end{array}$ & $\| \mathrm{la} / \mathrm{B}$ & $\begin{array}{c}60 \\
\text { pacientes }\end{array}$ & & $\begin{array}{l}\text { Duración de la } \\
\text { inyección y aplicación } \\
\text { local de frío seco }\end{array}$ & $\begin{array}{l}\text { Una duración de inyección de } 30 \text { s y } \\
5 \text { min de frío local (antes y después) } \\
\text { puede ser efectivo para disminuir el } \\
\text { dolor y la ocurrencia de } \\
\text { hematomas. }\end{array}$ \\
\hline LissowayJ & EUA & Inglés & $\begin{array}{l}\text { Reporte } \\
\text { de caso }\end{array}$ & $\mathrm{IV} / \mathrm{C}$ & $\begin{array}{c}1 \\
\text { paciente }\end{array}$ & IAM & $\begin{array}{l}\text { Hematoma } \\
\text { retroperitoneal }\end{array}$ & $\begin{array}{l}\text { La paciente falleció de un } \\
\text { hematoma retroperitoneal fatal. }\end{array}$ \\
\hline
\end{tabular}




\begin{tabular}{|c|c|c|c|c|c|c|c|c|}
\hline Autor & País & Idioma & Metodología & NE/GR & Muestra & Patología & Variables & Resultados \\
\hline Neviaser SA & EUA & Inglés & $\begin{array}{l}\text { Descriptivo/ } \\
\text { Observacional }\end{array}$ & $\mathrm{III/C}$ & $\begin{array}{c}135 \\
\text { pacientes }\end{array}$ & $\begin{array}{l}\text { THA primaria } \\
\text { O TKA }\end{array}$ & $\begin{array}{l}\text { Incidencia de } \\
\text { complicaciones } \\
\text { asociadas con el uso } \\
\text { de enoxaparina en } \\
\text { artroplastia }\end{array}$ & $\begin{array}{l}\text { El tratamiento postoperatorio en } \\
\text { pacientes sometidos a artroplastia } \\
\text { tiene un } 10 \% \text { de complicaciones } \\
\text { hemorrágicas mayores y } 27 \% \text { de } \\
\text { complicaciones menores. }\end{array}$ \\
\hline Crooke B & Australia & Inglés & $\begin{array}{l}\text { Reporte } \\
\text { de caso }\end{array}$ & $\mathrm{IV} / \mathrm{C}$ & $\begin{array}{c}1 \\
\text { paciente }\end{array}$ & $\begin{array}{l}\text { Embolia } \\
\text { pulmonar } \\
\text { bilateral }\end{array}$ & $\begin{array}{l}\text { Hematoma pélvico } \\
\text { extraperitoneal }\end{array}$ & $\begin{array}{l}\text { La escasez de tejido adiposo } \\
\text { subcutáneo y la consiguiente } \\
\text { superficialidad de la vaina del } \\
\text { músculo recto debe considerarse } \\
\text { para ubicaciones alternativas de } \\
\text { administración. }\end{array}$ \\
\hline Levin A & Israel & Inglés & $\begin{array}{l}\text { Descriptivo/ } \\
\text { Observacional }\end{array}$ & $\mathrm{III/C}$ & $\begin{array}{c}156 \\
\text { pacientes }\end{array}$ & & $\begin{array}{l}\text { Factores de riesgo de } \\
\text { sangrado en pacientes } \\
\text { ancianos }\end{array}$ & $\begin{array}{l}\text { El peso corporal bajo se asocia con } \\
\text { un mayor riesgo de sangrado a } \\
\text { pesar de una reducción en la dosis } \\
\text { según el peso. }\end{array}$ \\
\hline Despotovic N & Serbia & Inglés & $\begin{array}{l}\text { Descriptivo/ } \\
\text { Observacional }\end{array}$ & $\mathrm{III/C}$ & $\begin{array}{c}113 \\
\text { pacientes }\end{array}$ & IAM & $\begin{array}{l}\text { Relación riesgo/ } \\
\text { beneficio en pacientes } \\
\text { >75 años }\end{array}$ & $\begin{array}{l}\text { Aunque se aumentó la incidencia } \\
\text { de sangrado en pacientes con IAM } \\
\text { > } 75 \text { años con IR, la diferencia no } \\
\text { fue significativa. }\end{array}$ \\
\hline Singh $S$ & EUA & Inglés & $\begin{array}{l}\text { Reporte } \\
\text { de caso }\end{array}$ & $\mathrm{IV} / \mathrm{C}$ & $\begin{array}{c}1 \\
\text { paciente }\end{array}$ & $\begin{array}{l}\text { Embolia } \\
\text { pulmonar }\end{array}$ & Necrosis cutánea & $\begin{array}{l}\text { La utilidad de la HBPM se está } \\
\text { expandiendo rápidamente, es } \\
\text { imperativo que se estudien los } \\
\text { posibles efectos secundarios, } \\
\text { incluidas las reacciones cutáneas. }\end{array}$ \\
\hline Ozpolat B & Turquía & Inglés & $\begin{array}{l}\text { Reporte } \\
\text { de caso }\end{array}$ & $\mathrm{IV} / \mathrm{C}$ & $\begin{array}{c}1 \\
\text { paciente }\end{array}$ & TVP & $\begin{array}{l}\text { Hematoma } \\
\text { subpectoral en e } \\
\text { lado izquierdo }\end{array}$ & $\begin{array}{l}\text { La hemorragia mayor puede } \\
\text { ocurrir en pacientes ancianos con } \\
\text { enfermedad hepática crónica o } \\
\text { alteración de la función renal y, } \\
\text { que toman warfarina, aspirina, o } \\
\text { tienen terapia prolongada con } \\
\text { enoxaparina. }\end{array}$ \\
\hline Nourbakhsh E & EUA & Inglés & $\begin{array}{c}\text { De revisión } \\
\text { (sistematizada) }\end{array}$ & $\mathrm{IV} / \mathrm{C}$ & $\begin{array}{l}3 \\
\text { reportes de } \\
\text { casos }\end{array}$ & & & $\begin{array}{l}\text { El riesgo de hematomas es } \\
\text { mayor en mujeres mayores, con } \\
\text { anticoagulación concomitante o } \\
\text { medicamentos antiplaquetarios, } \\
\text { en personas con insuficiencia renal } \\
\text { y/o tensión abdominal (tos). }\end{array}$ \\
\hline Dadaeen A & Irán & Inglés & $\begin{array}{l}\text { Ensayo clínico } \\
\text { aleatorizado }\end{array}$ & $\mathrm{Ib} / \mathrm{A}$ & $\begin{array}{c}100 \\
\text { pacientes }\end{array}$ & & $\begin{array}{l}\text { Duración de la } \\
\text { inyección }\end{array}$ & $\begin{array}{l}\text { El aumento del tiempo de } 30 \\
\text { segundos redujo el alcance de } \\
\text { moretones y dolor. }\end{array}$ \\
\hline Dehghani K & Irón & Inglés & $\begin{array}{l}\text { Ensayo clínico } \\
\text { aleatorizado }\end{array}$ & $\mathrm{Ib} / \mathrm{A}$ & $\begin{array}{c}70 \\
\text { pacientes }\end{array}$ & SCA & $\begin{array}{l}\text { Duración de la } \\
\text { inyección }\end{array}$ & $\begin{array}{l}\text { La duración no tiene efecto en el } \\
\text { tamaño del hematoma, pero en } \\
\text { otros estudios, el sí puede ser } \\
\text { disminuido incrementando la } \\
\text { duración de la inyección. }\end{array}$ \\
\hline Yi L & China & Inglés & $\begin{array}{l}\text { De revisión } \\
\text { (sistematizada) }\end{array}$ & $\mathrm{IV} / \mathrm{C}$ & $\begin{array}{c}8 \\
\text { estudios }\end{array}$ & & $\begin{array}{l}\text { Duración de la } \\
\text { inyección }\end{array}$ & $\begin{array}{l}\text { La inyección lenta puede aliviar } \\
\text { y reducir el tiempo de dolor, la } \\
\text { aparición y disminución de } \\
\text { hematomas. }\end{array}$ \\
\hline
\end{tabular}

NE: nivel de evidencia, GR: grado de recomendación 
Cuadro 2. Evidencia relacionada con la administración de enoxaparina en adultos

\begin{tabular}{|c|c|c|c|c|c|c|}
\hline \multirow[t]{2}{*}{ Variables } & \multicolumn{5}{|c|}{ Bases de datos } & \\
\hline & CUIDEN & CINAHL & MEDLINE & LILACS & SciELO & SciELO \\
\hline Factores de riesgo $11,12,15,16,18,21-23$ & 1 & 3 & 1 & 1 & 2 & 0 \\
\hline Técnica de administración ${ }^{2438,49,50,51,52}$ & 6 & 8 & 2 & 0 & 0 & 3 \\
\hline Complicaciones ${ }^{13,14,17,19,20,39-49}$ & 0 & 6 & 9 & 0 & 1 & 0 \\
\hline
\end{tabular}

\section{DISCUSIÓN}

P ara utilizar de manera consciente, explícita y juiciosa la mejor evidencia clínica disponible y tomar las mejores decisiones sobre el cuidado de cada paciente ${ }^{53}$, se debe tener en cuenta la investigación realizada, la experiencia clínica, las preferencias y valores del paciente y los recursos disponibles. ${ }^{54}$ Sin embargo, poner en práctica los conocimientos encontrados en este trabajo, puede ser una limitante para llevar acabo la enfermería basada en evidencia, debido a que la mayoría de los artículos tienen un nivel de evidencia bajo (IV/C). Por otra parte, la procedencia de estos artículos es principalmente de países anglosajones, europeos y asiáticos, por lo que su aplicación en países latinoamericanos puede no ser similar y tener diferencias al momento de ponerlo en práctica. Además, de acuerdo a los resultados expuestos, se necesita publicación más reciente en este tema pues la mayoría de los artículos científicos encontrados tienen entre 4 y 10 años de haber sido publicados.

En los pacientes que son sometidos a tratamiento con HBPM, como la enoxaparina, la polifarmacia con fármacos que también actúan sobre la hemostasia, incrementan el riesgo de potencializar los efectos de este último, ${ }^{55}$ lo anterior puede estar explicado por el efecto sinérgico en cuanto a toxicidad que se obtiene con algunas combinaciones de fármacos ${ }^{56}$ poniendo en riesgo tanto su salud como su vida. La comprensión de ésta situación le permite al profesional de enfermería prever e inclusive evitar, en algunos casos, las reacciones adversas a la medicación por las interacciones medicamentosas. ${ }^{10}$

La administración subcutánea de HBPM tiene una biodisponibilidad cercana al 100\%, pero el fármaco se concentra, principalmente, en el plasma y en los tejidos muy vascularizados sin acceder casi al tejido graso ${ }^{14}$, por lo que se debe tener especial atención en los pacientes obesosdada, la menor proporción de masa magra, puede conducir a un exceso de anticoagulación y, por tanto, un mayor riesgo de hemorragia. ${ }^{57}$ Al igual que en las personas con insuficiencia renal, quienes tienen un efecto terapéutico mayor debido a una disminución del aclaramiento renal. ${ }^{174}$ Los pacientes de cardiología, tanto por sus características como por los tratamientos que reciben, suponen un grupo de riesgo para el desarrollo de complicaciones, por lo que se debe mantener una actitud expectante y cuidadosa en el momento de administrar tratamientos con HBPM en estos pacientes. ${ }^{17}$

El uso de jeringas precargadas, además de que evitan la reutilización del equipamiento y el contacto de los profesionales de salud con los materiales punzocortantes como las agujas que aumentan el riesgo de accidentes biológicos, potencialmente pueden beneficiar, también, a 
los pacientes que reciben tratamiento de HBPM, al reducirse el riesgo de hematoma comparado con una inyección subcutánea utilizando la aguja fija retráctil. Este hallazgo confirma que el uso del dispositivo de seguridad para aplicación de inyecciones subcutáneas es seguro para la utilización del paciente:,23,24 inclusive inyectar la burbuja de aire busca tres efectos: evitar la pérdida de medicamento, favorecer el sellado, y evitar medicación no deseada en capas más superficiales. ${ }^{26}$ La aspiración previa puede conducir a hematomas producidos por microdesgarros. ${ }^{59}$

El hematoma de la vaina del recto es un problema poco frecuente que ocurre principalmente en los pacientes que reciben tratamiento anticoagulante, pero puede ocurrir después del ejercicio, de latensión del abdomen, como tos crónica o estreñimiento, o incluso espontáneamente., Los posibles mecanismos subyacentes a este escenario pueden ser una inyección accidental de enoxaparina por vía intramuscular (recto abdominal), la interrupción de los vasos epigástricos debido a su gran anastomosis cerca de los sitios de inyección más comunes, así como su pronunciado efecto de la anticoagulación en los ancianos y en pacientes con trastornos hemorrágicos. ${ }^{18,47}$ La necrosis cutánea es una rara complicación de las inyecciones subcutáneas con heparina de bajo peso molecular. Se cree que la necrosis cutánea inducida por heparina es causada por una condición protrombótica local, mediada por anticuerpos, asociada con la activación plaquetaria y aumento de la producción de trombina. ${ }^{47}$

\section{CONCLUSIÓN}

$1 \begin{aligned} & \text { pesar de la limitante sobre el nivel de } \\ & \text { evidencia de los artículos se encontraron } \\ & \text { losfactores de riesgo máspredisponentes, } \\ & \text { las técnicas de administración más } \\ & \text { ade-cuadas y las complicaciones más }\end{aligned}$ frecuentes. Por lo que el cuidado enfermero, en la administración de enoxaparina, debe basarse en una adecuada valoración que permita identificar factores de riesgo y reducir la probabilidad de complicaciones.

En consideración al error causal, se justifica la realización de estudios de diseño más avanzados.

\section{8} Enf Neurol Vol. 16. No. 2 mayo - agosto 2017 


\section{REFERENCIAS BIBLIOGRÁFICAS}

1. Consejo de Salubridad General. Modelos de Seguridad del Paciente del Consejo de Salubridad General. Estándares para implementar el modelo en hospitales. Metas Internacionales de seguridad del paciente. $3 a$ ed México: 2015. 50-7 p. Disponible en: https://goo.gl/Bsrjg2

2. Vega SJ, Martínez RG, Goecke SH. Heparinas de bajo peso molecular en pacientes con enfermedad renal crónica ¿Es seguro su uso? Rev Med Chile (Internet). 2010 (citado 27 Mar 2017); 138: 487-95. Disponible en: https:// goo.gl/8G8Zc3

3. Opie LH, GershBJ. Fármacos para el corazón. bta ed. España: Mosby: 2006.

4. Pavón AF. Características de las heparinas de bajo peso molecular (HBPM). Emergencias (Internet). 2002 (citado 27 Mar 2017); 14: S38-S47. Disponible en: https://goo.gl/QYaczo

5. Trejo IC. Anticoagulantes: farmacología, mecanismos de acción y usos clínicos. CuadCir (Internet). 2004 (citado 27 de marzo de 2017); 18(1): 83-90. Disponible en: https://goo.gl/ISaalf

6. Whittemore R, Knafl K. The integrative review: updated methodology. J Adv Nurs. 2005 Dec; 52(5): 546-53

7. Agency for Healthcare Research and Quality (AHRQ).GPC Evidence Reports. (citado 29 Mar 2017). Disponible en: www.ahra.gov/clinic/ epcindexhtm\#metodology

8. Scottish Intercollegiate Guidelines Network.SIGN 50.A guidelines developer's handbook. (citado 29 Mar 2017). Disponible en: https://www. sign.ac.uk/pdf/sign50.pdf

9. Bardin L. Análise de conteúdo. 4 ed. Lisboa: Edições 70; 2008

10. Rangel CR, Rojas S, López M, Caviedes G. Prevalencia y factores asociados a la presencia de reacciones adversas a medicamentos en los pacientes tratados por medicina interna en el Hospital Universitario Hernando Moncaleano Perdomo. RevMed Risaralda (Internet). 2016 Mar (citado 29 Mar 2017); 22(2): 87-90. Disponible en: https://goo.gl/cJyNDr

11. Alcahúd CC, Iglesias MT, Lázaro CC, Córcoles JP, González AT, Lasema IF, et al. Administración de heparina de bajo peso molecular y aparición de complicaciones locales en pacientes de cardiología. Enferm Cardiol (Internet). 2009 (citado 29 Mar 2017); 16(47-48): 94-8. Disponible en: https://goo.gl/LBLbKV

12. Ozpolat B, Yazkan R, Aksoy M, DoDan S. Spontaneous pectoral hematoma secondary to enoxaparin for the treatment of deep venous thrombosis in an elderly man. Anadolu Kardivol Derg (Internet). 2008 Dec (citado 29 Mar 2017); 8(6): E-40. Disponible en: https://goo.gl/WasrHs

13. Nourbakhsh E, Anvari R, Nugent K. Abdominal wall hematomas associated with low-molecular-weight heparins: an important complication in older adults. J Am Geriatr Soc(Internet). 2011 Aug (citado 29 Mar 2017): 59(8): 1543-5. Disponible en: https://goo.gl/xonxiW

14. Manresa-Ramón N, Nájera-Pérez MD, Page-Del Pozo MA, SánchezMartínez I, Sánchez-Catalicio MM, Roldán-Schilling V. Establecimiento de un protocolo para el uso de la heparina en pacientes con características especiales. Farm Hosp (Internet). 2014 Abr (citado 29 Mar 2017); 38(2): 135 44. Disponible en: https://goo.gl/r7laqy

15. Schindewolf M, Schwaner S. Wolter M, Kroll H, Recke A, Kaufmann R, et al. Incidence and causes of heparin-induced skin lessons. CMAJ (Internet). 2009 Oct (citado 29 Mar 2017); 12(8): 7-5. Disponible en: https://goo.gl//4b8LO
16. Kayrak M Bacaksiz A Yazici M Is enoxaparin injection from the abdominal wall safe in elderly people?: a fatal case of rectus sheath hematoma. Can Fam Physician (Internet). 2008 Sep (citado 29 Mar 2017); 54 1246-48. Disponible en: https://goo.gl/oLfFBu

17. Despotovic N, Erceg P, Nikolic-Despotovic M, Milosevic DP, Davidovic M. Bleeding during enoxaparin treatment more common with age over 75 years and severe renal insufficiency. Drugs Aging (Internet). 2007 (citado 29 Mar 2017); 24(9): 777-9. Disponible en: https://goo.gl/aV5kfL

18. Yusuf A, Levent Ö, Dhsan Ü, Leyla YA. A salientrectusabdominis hematoma duetoenoxaparin. Anatol J Cardiol (Internet). 2007 (citado 29 Mar 2017); 7(1) 92. Disponible en: https://goo.gl/kLwbho

19. Crooke B, Callaway LK. Fatal haemorrhage associated with enoxaparin Intern Med J (Internet). 2007 Mar (citado 29 Mar 2017); 37(3): 207-8. Disponible en: https://goo.gl/VHL1Dd

20. Levin A, Ben-Arzi M, Beckerman P, Haber G, Varon D, Ben-Yehuda A, et al. Factors associated with bleeding in elderly hospitalized patients treated with enoxaparin sodium: a prospective, open-label, observational study. DrugsAging (Internet). 2009 (citado 29 Mar 2017); 26(1): 77-85. Disponible en: https://goo.gl/Py5im8

21. Gomes M, Ramacciotti E, Litinas E, Fareed J. Consideraciones sobre e uso de enoxaparina genérica en SCA. Ara Bras Cardiol (Internet). 2010 Oct (citado 29 Mar 2017); 95(4): 551-2. Disponible en: https://goo.gl/W2pclu

22. Neviaser SA, Chang C, Lyman S, Gonzales A, Haas BS. High incidence of complications from enoxaparin treatment after arthroplasty. Clin Orthop Relat Res (Internet). 2010 Jan (citado 29 Mar 2017); 468(1): 115-9. Disponible en: hitps://goo.gl/NrkUOJ

23. Lamblet LCR, Meira ES, Torres S, Ferreira BC, Martucchi SD. Ensavo clínico aleatorio para evaluación del dolor y hematoma durante la administración de medicamentos por vía subcutánea e intramuscular: ¿es necesario cambiar las aqujas? Rev Latino-Am Enfermagem (Internet). 2011 Sep-Oct (citado 29 Mar 2017); 19(5): (10 pantallas). Disponible en: https://goo.gl/1 mVitv

24. Gómez MJ, Martínez MA, García l. ¿Cuál es la técnica idónea para disminuir las complicaciones locales secundarias a la administración subcutánea de enoxaparina? Ensayo clínico aleatorizado. Enferm Clin (Internet). 2005 (citado 29 Mar 2017); 15(6): 329-34. Disponible en: https://goo.gl/NrSWPe

24. Cerrillo-Patiño JR. Administración de heparinas de bajo peso molecular a mayor experiencia, peores resultados. La jeringa precargada mejora los resultados auto-referidos de la técnica de administración. Evidentia (Internet) 2007 Ene-Feb (citado 29 Mar 2017); 4(13). Disponible en: https://goo.gl/e5fo6E

26. Tomás-Rojas J, Cachinero-Nieto M, Tomás-Rojas I, García-La Fuente F, Sánchez García A. Ferrándiz-Viñolo JA. Para administración de medicación subcutánea o intramuscular, ise purga el aire en las jeringas precargadas? Garnata (Internet) 2017 (citado 29 Mar 2017); 21: 43-7. Disponible en: https://goo.gl/lgxfna

27. Rodríguez-Gallego I, Quijano-Campos JC, Piñeiro-Rojas R, Rodríguez Pappalardo F, Granados-Matute E, Moreno-Gordillo JM. Técnicas de administración de heparina de bajo peso molecular. Rev Paraninfo Digito (Internet). 2011 (citado 29 Mar 2017); 12(5). Disponible en: https://goo.gl/glbgmz

28. Palese A, Aidone E, Dante A, Pea F. Occurrence and extent of bruising according to duration of administration of subcutaneous low-molecularweight heparin. A quasi-experimental case-crossover study. J Cardiovaso Nurs (Internet). 2013 Sep-Oct (citado 29 Mar 2017); 28(5): 473-82. Disponible en: https://goo.gl/W8Zob 
29. Visvanathan $V$. Slow vs fast subcutaneous heparin injections for prevention of pain and bruising. A.JN (Internet). 2015 Dec (citado 29 Mar 2017); 115(12): 27. Disponible en: https://goo.gl/Dvnx8a

30. Uzun S, Aciksoz S, Arslan F, Yildiz C, Akyol M. The effect of administration protocol of subcutaneous enoxaparin injection on formation of ecchymosis Orthopaedic Nursing (Internet).2016 Mar-Apr (citado 29 Mar 2017); 35(2) 120-5. Disponible en: https://goo.gl/76uBrr

31. Pourghaznein T, Azimi AV, Jafarabadi MA. The effect of injection duration and iniection site on pain and bruising of subcutaneous iniection of heparin. J Clin Nurs (Internet). 2013 Jun (citado 29 Mar 2017); 17(23): 1105-13. Disponible en: https://goo.gl/Ekt8gE

32. Zaybak A, Khorshid L. A study on the effect of the duration of subcutaneous heparin injection on bruising and pain. J Clin Nurs (Internet). 2008 Feb (citado 29 Mar 2017); 17(3): 378-85. Disponible en: https://goo.gl/xSnMR6

33. Sendir M, Büyükyilmaz F, Celik Z, Taskopru I. Comparison of 3 methods to prevent pain and bruising after subcutaneous heparin administration. Clin Nurse Spec (Internet). 2015 May-Jun (citado 29 Mar 2017); 29(3): 123-88. Disponible en: hittps://goo.gl/xAygUe

34. Dadaeen A, Bahreini M, Bazi P, Ostovar A, Raeisi A, Dobaradaran S. The effect of duration of subcutaneous injection on the extent of bruising and pain intensity at injection sites among patients receiving enoxaparin sodium a randomized self-controlled clinical trial. Int Cardiovas Res J (Internet). 2015 (citado 29 Mar 2017); 9(2): 77-82. Disponible en: https://goo.gl/eOVmWn

35. Yi L, Shuai T, Tian X, Zeng Z, Ma L, Song $G$. The effect of subcutaneous injection duration on patients receiving low-molecular-weight heparin: evidence from a systematic review. International Journal of Nursing Sciences (Internet) 2016 Apr (citado 29 Mar 2017); 3(1): 79-88. Disponible en: https://goo.gl/76uBrr

36. Amaniyan S, Varaei S, Vaismoradi M, Haghani H, Sieloff C. Effect of local cold and hot pack on the bruising of enoxaparin sodium injection site: a randomized controlled trial. Contemp Nurse (Internet). 2016 Feb (citado 29 Mar 2017); 52(1): 1-12. Disponible en: https://goo.gl/C8Q07r

37. Balci AR. The effect of local dry heat pack application on recovering the bruising associated with the subcutaneous injections of heparin. J Clin Nurs (Internet). 2013 Sep (citado 29 Mar 2017); 22(17-18): 2537-5. Disponible en: https://goo.gl/efxQbl

38. Pierri H, Rodrigues GHP. Sangramento abdominal com heparina de baixo peso molecular. Rev Assoc Med Bras (Internet). 2005 Feb (citado 29 Mar 2017); 51 (1): 10.Disponible en: https://goo.gl/CcbKyw

39. Firoozbakhsh S, Parsaei R, Jafarshad R. Hematoma of rectus sheath following subcutaneous enoxaparin injection. Acta Med Iran (Internet). 2013 Jan (citado 29 Mar 2017); 51 (5): 334-6. Disponible en: https://goo.gl/FDfCCA

40. Rahman MS, Soo L, Qasim A. An unusual case of massive subcutaneous chest wall haemorrhage with enoxaparin. Acute Card Care (Internet). 2017 Sep (citado 29 Mar 2017); 13(3): 199-201. Disponible en: https://goo.gl/h5XVY5

41. Parkinson F, Khalid U, Woolgar J. Rectus sheath haematoma: a serious complication of a commonly administered drug. BMJ (Internet). 2013 Apr (citado 29 Mar 2017). Disponible en: https://goo.gl/8N3CDs

42. Loidi PL Valcayo PA, Ruíz de Azúa CAY Yanguas BI. Dermatosis ampollosa hemorrágica a distancia inducida por heparina: descripción de 2 nuevos casos. Cutan Ocul Toxicol (Internet). 2016 (citado 29 Mar 2017); 35(2): 516-7. Disponible en: https://goo.gl/jMMKvW

43. Sobieszczanska M, Tubek S, Kura I. Pyodermagan grenosum-like skin changes after subcutaneous administration of low molecular weight heparin. Hum Vaccinlmmun other (Internet). 2014 (citado 29 Mar 2017); 10(4): 968-9.Disponible en: https://goo.gl/NiH7Ua
44. Ivanova I, SegarraEM, Corredor JA, Pagés-Reverter JM. Delayed hypersensitivity reaction due to subcutaneous sodium enoxaparin Semergen (Internet). 2013 Jan-Feb (citado 29 Mar 2017); 39(1): 59-60. Disponible en: https://goo.gl/u7bpCX

45. Kumar DA. Low-molecular-weight heparin-associated fat necrosis of the breast.Age Ageing (Internet). 2005 Mar (citado 29 Mar 2017); 34(2): 193-4. Disponible en: https://goo.gl/gEpN8T

46. Lissoway J, Booth A. Fatal retroperitoneal hematoma after enoxaparin administration in a patient with paroxysmal atrial flutter. Am J Health-Sys Pharm (Internet). 2010 May (citado 29 Mar 2017); 67(10): 806-9. Disponible en hittps://goo.gl/OVyEMW

47. Toll A, Gallardo F, Abella E, Fontcuberta J, Barranco C, Pujol RM. Low molecular-weight heparin-induced skin necrosis: a potential association with pre-existent hypercoagulable states. Int J Dermato (Internet). 2005 Nov (citado 29 Mar 2017); 44(17): 964-6. Disponible en: https://goo.gl/x6RcZ8

48. Singh $S$, Verma $M$, Bahekar $A$, Agrawal $P$, Duggal J, lliescu $M$, et al Enoxaparin-inducedskin necrosis: a fatal outcome. Am J Ther (Internet). 2007 Jul-Aug (citado 29 Mar 2017); 14(4): 408-10. Disponible en: https://goo.gl/DATjC7

49. Cerrillo-Patiño JR. La enoxaparina subcutánea precargada reduce las complicaciones locales de administración. Evidentia (Internet). 2007 EneFeb (citado 29 Mar 2017); 4(13). Disponible en: https://goo.gl/40xLCl

50. Black L. Ditch the pinch: bilateral exposure injuries during subcutaneous injection. Am J Infect Control (Internet). 2013 Sep (citado 29 Mar 2017); 41 (9) 815-9. Disponible en: https://goo.gl/TzPKSt

51. Rushing J. Administering an enoxaparin injection. Nursing (Internet). 2008 Mar (citado 29 Mar 2017); 38(3): 19. Disponible en: https://goo.gl/eUISZS

52. Dehghani K, Najari Z, Dehghani H. Effect of subcutaneous enoxaparin injection duration on bruising size in acute coronary syndrome patients. Iran J Nurs Midwifery Res (Internet). 2014 Nov (citado 29 Mar 2017); 19(6): 564-8. Disponible en: https://goo.gl//OIXZP

53. Sackett DL, Rosenberg WMC, Muir JA, Haynes RB, Richardson WS Evidence Baed Medicine: What it is and what it isnt. Br Med J 1996: 312: 71-2.

54. Gálvez TA. Enfermería basada en la evidencia. Cómo incorporar la investigación a la práctica de los cuidados. México: Fundación INDEX. 2001

55. Casamitjana CN. Anticoagulantes inyectables. Heparinas. Farmacia Profesional (Internet). 2001 May (citado 29 Mar 2017); 15(5): 70-3. Disponible en: https://goo.gl/Zthzyl

56. Routledge PA, O'Mahony MS. Woodhouse KW.Adverse drug reactions in elderly patients. Br J Clin Pharmacol (Internet). 2004 Feb (citado 29 Mar 2017) 57(2): 127-6. Disponible en: https://goo.gl/b3z57

57. Richard AA, Kim S, Moffett BS, Bomgaars L, Mahoney JD, Yee DL Comparison of Anti-XaLevels in Obese and Non-Obese Pediatric Patients Receiving Treatment Doses of Enoxaparin. J Pediatr (Internet). 2013 Fel (citado 29 Mar 2017); 162(2): 293-6. Disponible en: hittps://goo.gl/TsEiZq

58. Hutin Y, Hauri A, Chiarello L, Catlin M, Stilwell B, Ghebrehiwet T, et. al. Best infection control practices for intradermal, subcutaneous and intramuscular needle injections. Bull World Health Organ (Internet). 2003 (citado 29 Mar 2017); 81 (7): 497-500. Disponible en: https://goo.gl/JKhgpx

59. Garrido IM, López L, Seda J. Heparinas de bajo peso molecular Cambios en la técnica de administración. Rev Rol Enferm. 1996; 217: 55-8.

60. Cherry WB, Mueller PS. Rectus sheath hematoma. Review of 126 cases a a single institution. Medicine (Baltimore) (Internet). 2006 Mar (citado 29 Mar 2017); 85(2): 105-10. Disponible en: https://goo.gl/5gkZWf
120 nMN MWhat Enf Neurol Vol. 16. No. 2 mayo - agosto 2017 